\title{
A One-step, Versatile Synthesis of Dibenzo [n.2.2] Macrobicyclic Compounds via a Conformation- Directed Macrocyclization Reaction
}

\section{Rubén Lobato, Alberte X. Veiga, Jaime Pérez-Vázquez, Fernando}

Fernández-Nieto, M. Rita Paleo, ${ }^{*}$ and F. Javier Sardina*

Departamento de Química Orgánica and Centro Singular de Investigación en Química Biológica y Materiales Moleculares. Universidad de Santiago de Compostela, 15782

Santiago de Compostela, Spain

\section{rita.paleo@usc.es; javier.sardina@usc.es}

Received Date (will be automatically inserted after manuscript is accepted)

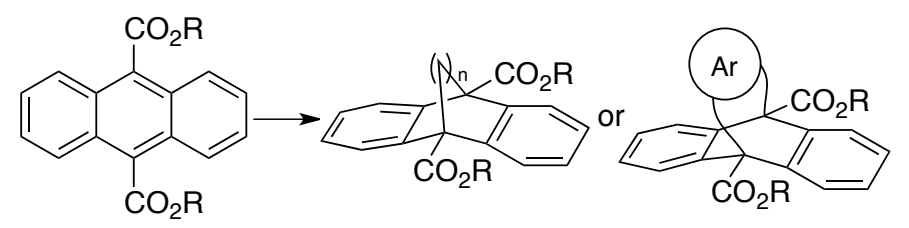

A series of dibenzo [n.2.2] bicyclic compounds $(n=2-20)$ were prepared in one step and good yields starting from dimethyl anthracene-9,10-dicarboxylate. Reduction of the aromatic diester using lithium/naphthalene led to a bisenolate that was cyclized with a variety of bis-electrophiles. The ease of the cyclization is probably due to the puckered conformation of the intermediate formed after the first alkylation step, in which the newly introduced chain that will become the bridge portion occupies a pseudoaxial position, positioning the leaving group close to the enolate nucleophile in the macrocyclization step. 
From a synthetic point of view the preparation of medium- and large-sized carbocycles is not a trivial task. The numerous strategies envisioned for the construction of these types of meso- and macrocycles can be grouped in three general approaches: cycloadditions, cyclization reactions, and transformations of pre-existing rings (fragmentation of bicycles and ring expansion reactions).

Medium-sized rings ${ }^{1}$ are especially hard to prepare efficiently by conventional cyclization approaches due to both disfavoring entropic and enthalpic factors. The ease (or lack thereof) of their preparation has been shown to be very sensitive to changes in the makeup of the ring (presence of heteroatoms and substituents). Ionic and radical fragmentation processes, as well as ring-expansion reactions, ${ }^{2}$ have been used for the preparation of 7- to 10 membered rings. Ring closing olefin metathesis (RCM) on the other hand, has been successfully applied for the synthesis of medium-sized rings in systems that present some sort of conformational constrain (such as additional rings or stereoelectronic effects), as well as for the synthesis of macrocycles where the presence of a polar functional group has shown to be more important than ring size for the success of the reaction. ${ }^{3}$

Macrocyclization methods ${ }^{4}$ include carbocyclization, ${ }^{5}$ macrolactonization $^{6}$ and macrolactamization ${ }^{7}$ reactions. Probably the most general and direct approach for the preparation of macrocycles consists in the ring-closure reaction $^{8}$ of a bifunctional flexible chain possessing a nucleophile and an electrophile at each end, although this is usually a low yielding process since polymerization can be an efficient competing reaction. That is the reason why the majority of the synthetic approaches for the preparation of macrocycles usually require high dilution conditions. ${ }^{9}$ Alternative strategies to improve the yield of the macrocyclization step basically rely on the incorporation of a rigid group to restrict rotation in the chain, on some kind of conformational pre-organization ${ }^{10}$ or, more often, on the presence of a metal ion around which the molecule preorganizes the two reacting centers in a productive conformation (the template effect). ${ }^{11}$

We describe herein the preparation of different dibenzo [n.2.2] bicyclic compounds, where $n$ varies from 2 to 20 , usually in good yields, almost regardless of the ring size (small, medium or large), starting from an anthracene dicarboxylate. No high dilution conditions are required, neither a template effect nor the presence of a rigid group appear to be responsible for the efficiency of this transformation.

We have previously reported ${ }^{12}$ the successful coupling of a dearomatization reaction of aromatic and heteroaromatic diesters with a bis-alkylation reaction to prepare fused $[6,5],[6,6]$, and [6,7] bicyclic compounds. The reaction was applied to benzene, pyridine, naphthalene, furane, thiophene and benzofurane dicarboxylates. Mechanistically, the reaction is initiated by anionic tin nucleophiles to give, via a stanna-Brook rearrangement, a bis-enolate intermediate that can be easily alkylated and cyclized by reaction with different bis-electrophiles. In some cases these bis-alkylative cyclizations could also be performed with sodium metal as a substitute for the tin anions (for the generation of the intermediate bis-enolates). We now disclose that this type of cyclizations also occurs with anthracene derivatives substituted by carboxylate groups at positions 9 and 10 , but in this case the products are not fused, but bridged bicyclic compounds instead. When diisopropyl anthracene-9,10-dicarboxylate (1a) was treated with $\mathrm{Me}_{3} \mathrm{SnLi}$ in $\mathrm{THF}$ at $-78{ }^{\circ} \mathrm{C}$ followed by 1,3dibromopropane, the crude reaction ${ }^{1} \mathrm{H}-\mathrm{NMR}$ showed the presence of only one product, identified as the bridged bicyclic compound $\mathbf{2 a}$, which could be isolated in $92 \%$ yield (see Scheme 1). This encouraging result prompted us to explore the scope of this reaction with different electrophiles. When the bis-alkylation of the intermediate bis-enolate was carried out with 1,4-dibromobutane or cis-1,4-dichlorobutene the bridged bicyclic compounds 3a and 12a were isolated in $53 \%$ and $92 \%$ yield, respectively.

Although we had established over the years that tin compounds are very useful reagents in dearomatizationbis-alkylation reactions, their inherent toxicity and high cost are drawbacks serious enough to compel us to find alternative reagents for these transformations.

As anthracene dicarboxylate $\mathbf{1}$ is an electron deficient aromatic molecule, we considered that it could be reduced under modified (ammonia-free) Birch ${ }^{13}$ conditions, and if the reaction were carried out in the absence of a proton source, a bis-enolate should be obtained as the reaction intermediate, which could then be efficiently bisalkylated. To this end we chose lithium as the metal and naphthalene as the electron carrier. ${ }^{14}$ In practice, the reaction of dimethyl anthracene-9,10-dicarboxylate (1b) with excess lithium and naphthalene (ca 5:1) in THF, followed by 1,3-dibromopropane gave the bridged bicyclic compound $\mathbf{2 b}$ in $95 \%$ yield (see Scheme 1).

We then decided to study the ability of this dianionic system to react with different electrophiles to give rise to bicyclic rings of different bridge sizes. When the solution obtained by reduction of anthracene diester $\mathbf{1 b}$ was quenched with 1,4-dibromobutane, the [4.2.2] bridged compound $\mathbf{3 b}$ was obtained in $52 \%$ yield, a very good yield based on the difficulty in forming rings of this particular size (8-membered ring). However, when 1,5dibromopentane was used as the electrophile we could not isolate the corresponding 9-membered ring under any of the reaction conditions tested. At low temperature a mixture of mono- and dialkylated (1 electrophile coupled with 2 nucleophiles) products, and dihydroanthracene9,10-dicarboxylate was obtained, while the bisalkylated (1 nucleophile alkylated with 2 electrophiles) product was isolated at higher temperatures. No cyclized product was observed when the monoalkylated product was isolated and then treated with LDA. Although disappointing, this was not a very surprising result as it is well known that the ease of carbocyclization depends on the ring size, with 8-and 9-membered rings being the most difficult to form.

Better results were obtained when bis-electrophiles with longer chains were used. Thus, with 1,6- 
Scheme 1. Synthesis of bridged [n.2.2] bicyclic compounds<smiles>[R]OC(=O)c1c2ccccc2c(C(=O)OC(C)(C)C)c2ccccc12</smiles>

1a, $\mathrm{R}=\mathrm{iPr}$

2a-3a, $\mathrm{R}=\mathrm{iPr}$

1b, $\mathrm{R}=\mathrm{Me}$

$2 b-11 b, R=M e$

$13 b-16 b$

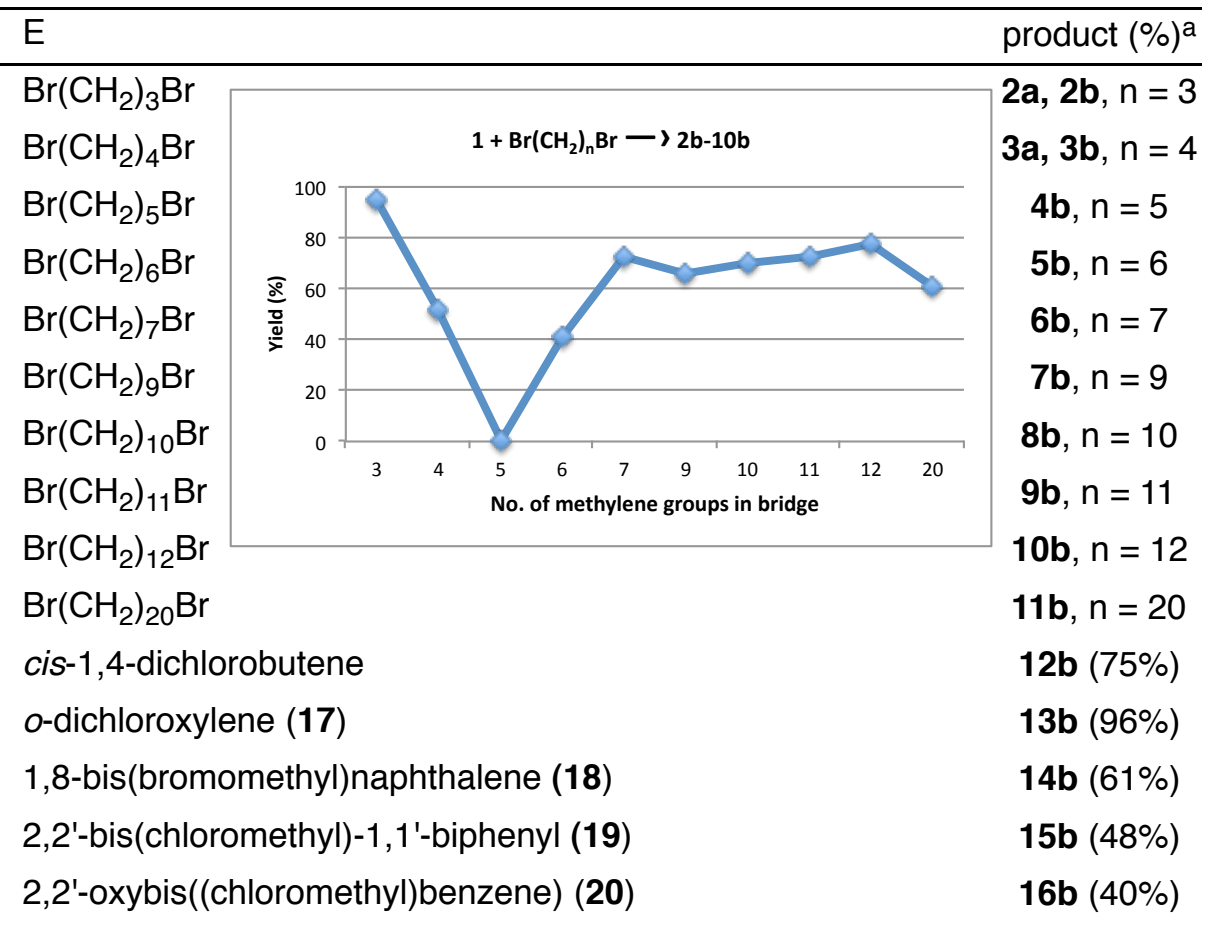

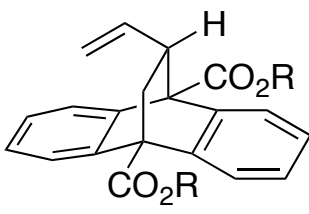

12a, $\mathrm{R}=i \mathrm{Pr}$

$12 b, R=M e$

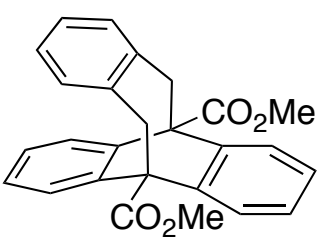

$13 b$

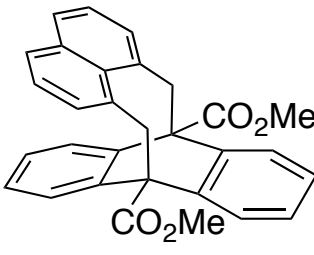

$14 \mathrm{~b}$

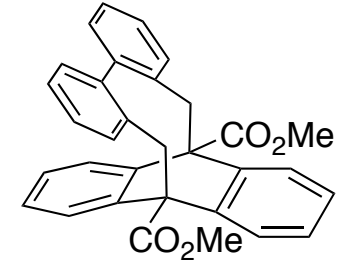

$15 b$

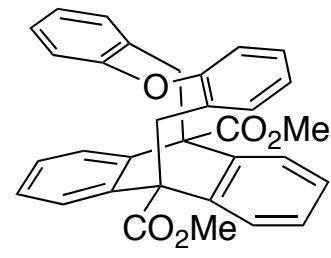

16b

${ }^{\text {a) }}$ Yields with aliphatic bis-electrophiles: 2b, 95\%; 3b, 52\%; 4b, 0\%; 5b, 41\%; 6b, 72\%; 7b, 66\%; 8b, 70\%; 9b, 72\%; 10b, 77\%; 11b, 61\%.

The synthesis of macrocyclic compounds was shown to be very easy for this system as 1,9-dibromononane allowed the preparation of bridged bicyclo [9.2.2] compound $\mathbf{7 b}$ in $66 \%$ yield (13-membered ring), 1,10dibromodecane yielded [10.2.2] bicyclic compound $\mathbf{8 b}$ in $70 \%$ yield (14-membered ring), and 1,11dibromoundecane and 1,12-dibromododecane led to the corresponding macrocycles $\mathbf{9 b}$ and $\mathbf{1 0 b}$ in 72 and $77 \%$ yield, respectively. Based on these results, it seemed as though macrocycles of almost any size could be prepared in high yield just by increasing the length of the methylene chain of the electrophile. To test this assertion we decided to prepare a 24-membered ring using the corresponding bis-electrophile, 1,20-dibromoicosane (the

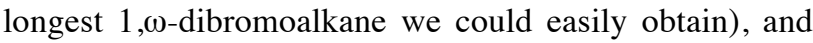
thus [20.2.2] bicyclic compound 11b was isolated in $61 \%$ yield. What is remarkable of this reaction is that there is no need for using high dilution conditions to obtain the macrocycles. ${ }^{15}$ The trend observed for the propensity of the monoalkylated intermediates to cyclize (measured as the yield of the bridged product as a function of bridge size) (Scheme 1) roughly follows that observed by 
Illuminati et al. in their seminal kinetic study on macrocyclizations. ${ }^{8 d}$ As can be seen from the plot in Scheme 1, the ease of the cyclization goes through a minimum while trying to close a 9-membered ring (closing 8 or 10-membered rings is difficult but possible), a behaviour clearly rooted in the importance of ring-strain factors in these types of macrocyclizations. ${ }^{8 b}$ To nullify the efect of this factor we decided to use bis-electrophiles that incorporate a rigid group to restrict rotation in the chain (17-20) in an attempt to favor the formation of medium sized rings. An excellent result was obtained when using $o$-dichloroxylene (17), as the 8-membered ring 13b was isolated in $96 \%$ yield. The 9-membered ring 14b could also be prepared in $61 \%$ yield when 1,8 bis(bromomethyl)naphthalene (18) was used as the electrophile. The corresponding 10- and 11-membered rings $\mathbf{1 5 b}$ and $\mathbf{1 6 b}$ were isolated in moderate yields when 2,2'-bis(chloromethyl)-1,1'-biphenyl (48\%) and 2,2-oxibis[(chloromethyl)benzene] $(40 \%)$ were used as biselectrophiles, respectively. In these last two examples the lower yields of the bridged products do not appear to arise from difficulties in the cyclization step, but from a competing halogenation of the intermediate bis-enolates followed by rearomatization to the starting anthracene, which accounts for the balance of material in these reactions.

Scheme 2. Reactive conformations of the enolate intermediate

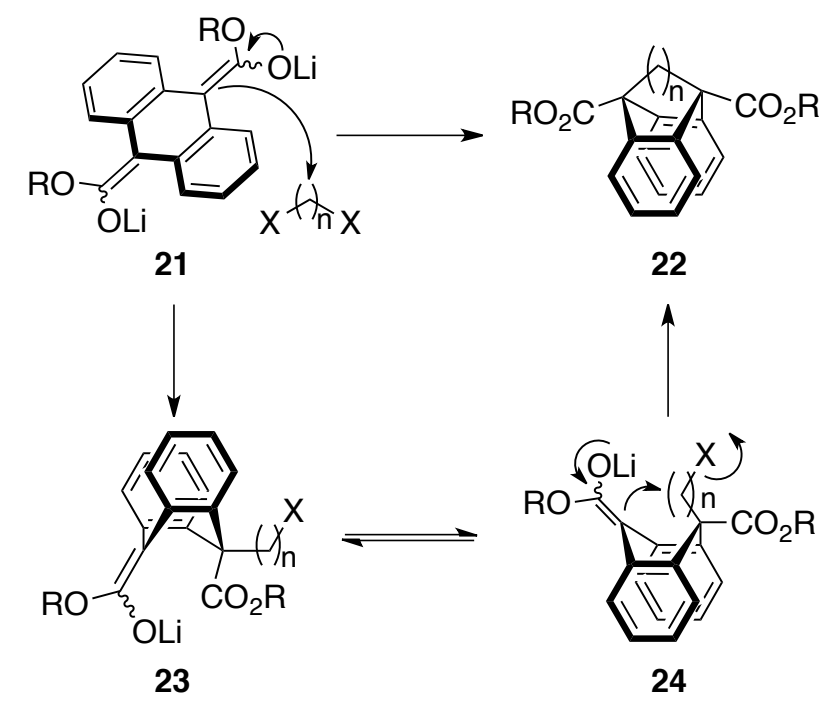

To explain the ease of this meso- and macrocyclization, almost independent of the ring size of the product, we have to consider the reactive conformation of the monoalkylated intermediate (see Scheme 2). Numerous studies ${ }^{16}$ concerning the conformational analysis of 9-alkyl-9,10-dihydroanthracenes have shown the existence of a favored non planar conformation, with departure from planarity increasing with the bulkiness of the substituent. Moreover, it has been demonstrated that in the preferred conformation, the substituent occupies a pseudoaxial position in a more or less flattened, boatshaped conformation. ${ }^{17}$ We propose that the distinctive facilitating element in this newly described cyclization is the conformation of the intermediate monoenolate (see Scheme 2): a boat conformation with the alkyl chain bearing the leaving group disposed in a pseudoaxial position (in close proximity to the nucleophillic carbon of the enolate), which greatly facilitates the intramolecular alkylation.

To check this conformational hypothesis we prepared a model system of the key monoenolate intermediate (by reaction of dimethyl 9-propyl-9,10-dihydroanthracene9,10-dicarboxylate, 25, with LDA) and studied its structure and conformation by NMR (Scheme 3). ${ }^{18}{ }^{1} \mathrm{H}-$ NMR spectrum of propylated diester 25 , in $\mathrm{THF}_{-} \mathrm{D}_{8}$ at $78{ }^{\circ} \mathrm{C}$, shows the presence of a singlet at $5.30 \mathrm{ppm}$ due to the $\mathrm{C}-10$ proton, and a multiplet at $2.15 \mathrm{ppm}$ due to the $\mathrm{CH}_{2}$ of the propyl group bound at C-9. When a solution of 25 in deuterated THF was added to an NMR tube containing a solution of LDA $(210 \mathrm{~mol} \%)$ at $-78{ }^{\circ} \mathrm{C}$, we observed the disappearance of the signal at $\delta 5.30 \mathrm{ppm}$ in less than $30 \mathrm{~min}$, and the appearance of signals corresponding to the enolate (see Supporting Information and selected peaks in Scheme 3).

Scheme 3. NMR data for monoenolate $\mathbf{2 6}$ ( $\delta$ in ppm)

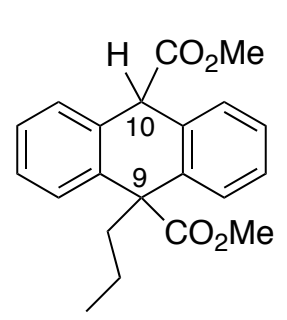

25

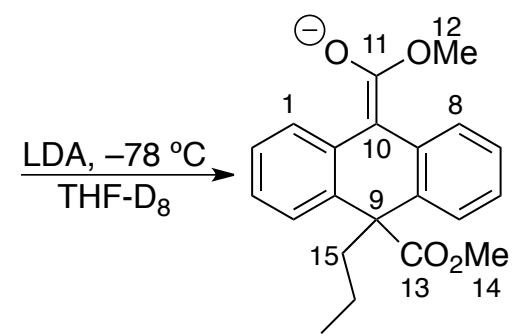

26

\begin{tabular}{cccccccccc}
\hline $\mathbf{2 6}$ & 1 & 8 & 9 & 10 & 11 & 12 & 13 & 14 & 15 \\
\hline${ }^{1} \mathrm{H}-\mathrm{NMR}$ & 8.14 & 7.84 & & & & 3.54 & & 3.61 & 1.57 \\
$\Delta \delta^{\mathrm{a}}$ & 0.70 & 0.40 & & & & 0.03 & & 0.2 & -0.58 \\
${ }^{13} \mathrm{C}-\mathrm{NMR}$ & 126.4 & 127.4 & 59.5 & 80.9 & 165.2 & 49.4 & 177.2 & 51.9 & 41.3 \\
$\Delta \delta^{\mathrm{a}}$ & -3.7 & -2.7 & 3.5 & 30.7 & -8.1 & -3.6 & 1.1 & -0.9 & -4.8
\end{tabular}

(a) $\Delta \delta=\delta$ enolate $26-\delta$ protonated 25
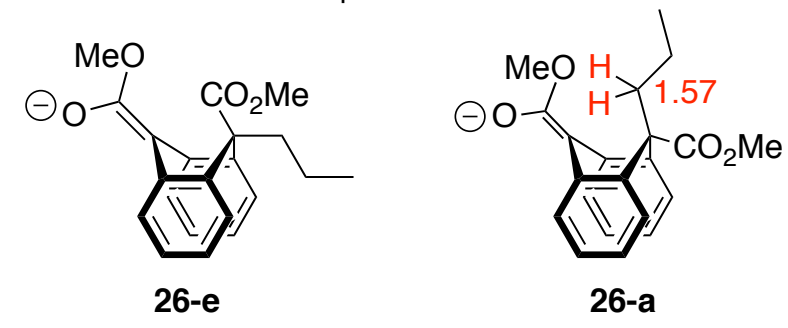

An NMR study of the deprotonation of 9-carboethoxy9,10-dihydroanthracene performed by Rabideau's group ${ }^{19}$ pointed out that the enolate is the preferred structure for 
the monoanion and thus the carbonyl $\pi$ overlap forces some folding of the central ring. If the central ring adopts a boat conformation, as suggested by Rabideau, the chemical shift of the group in pseudoaxial should be affected by the cone-shape shielding zone of the enolate C-C double bond. Thus, if the propyl group preferentially exists in the pseudoaxial position, a sizeable upfield displacement of the ${ }^{1} \mathrm{H}-\mathrm{NMR}$ signal should be observed (26-a, see Scheme 3). However, such a displacement should not be observed if the orientation of the propyl group in the enolate were preferentially pseudoequatorial, 26-e. As can be seen in Scheme 3, the methylene group closest to the ester moiety experiences a considerable upfield shift $(\Delta \delta=-0.58 \mathrm{ppm})$, a value in agreement with the proposed boat conformation for the enolate and the pseudoaxial disposition for the propyl group. These observations lend credence to our proposal that puckered conformation lies at the heart of the ease of this cyclization.

In conclusion, we have developed a highly efficient synthesis of bridged [n.2.2] bicyclic compounds based on the reductive alkylation of dimethyl anthracene-9,10dicarboxylate with lithium and naphthalene. Under these conditions a stable bis-enolate was generated and then cyclized by reaction with a variety of bis-electrophiles, allowing the one-pot preparation of medium and largesized dibenzobicycles.

Bridgehead-substituted dibenzo [2.2.2] compounds have been used for the design and study of molecular devices. ${ }^{20}$ We believe our approach could open the way for the preparation of analogous systems with more varied bicyclic cores. We are currently exploring further applications of this conformationally directed macrocyclization methodology towards this end.

Acknowledgment This work was supported by the Ministerio de Economía y Competitividad of Spain (CTQ2011-22436) and Xunta de Galicia (PGIDIT10PXIB209113PR, 10PXIB209155PR and 2007/085).

Supporting Information Available. Full experimental procedures, characterization data, and NMR spectra for all new compounds. This material is available free of charge via the Internet at http://pubs.acs.org.

1. (a) Watson, I. D. G ; Ritter, S ; Toste, F. D. J. Am. Chem Soc 2009, 131, 2056-2057. (b) Yet, L. Chem. Rev. 2000, 100, 2963-3007. (c) Mehta, G.; Singh, V. Chem. Rev. 1999, 99, 881-930. (d) Murakami, M. Angew. Chem. Int. Ed. 2003, 42, 718-720. (e) Yu, Z.-X.; Wang, Y.; Wang, Y. Chem. Asian J. 2010, 5, 1072-1088.

2. (a) Nubbemeyer, U. Eur. J. Org. Chem. 2011, 1801-1816. (b) Yet, L. Tetrahedron 1999, 55, 9349-9403. (c) Molander, G. A. Acc. Chem. Res. 1998, 31, 603-609.

3. (a) Fürstner, A. Top. Catal. 1997, 4, 285-299. (b) Maier, M. E. Angew. Chem. Int. Ed. 2000, 39, 2073-2077. (c) Nicolaou, K. C.; Bulger, P. G.; Sarlah, D. Angew. Chem. Int. Ed. 2005, 44, 4490-4527. (d) Gradillas, A.; Pérez-Castells, J. Angew. Chem. Int. Ed. 2006, 45, 6086-6101. (e) Prunet, J. Eur. J. Org. Chem. 2011, 3634-3647.

4. (a) Meng, Q.; Hesse, M. Top. Curr. Chem. 1992, 161, 107-176. (b) Wessjohann, L. A.; Ruijter, E. Top. Curr. Chem. 2005, 243, 137-184. (c) Wessjohann, L. A.; Rivera, D. G.; Vercillo, O. E. Chem. Rev. 2009, 109, 796-814. (d) Crane, E. A.; Scheidt, K. A. Angew. Chem. Int. Ed. 2010, 49, 8316-8326. (e) Obradors, C.; Leboeuf, D.; Aydin, J.; Echavarren, A. M. Org. Lett. 2013, 15, 1576-1579.
5. (a) Casadei, M. A.; Galli, C. ; Mandolini, L. J. Am. Chem. Soc. 1984, 106, 1051-1056. (b) Nicolaou, K. C.; Bulger, P. G.; Sarlah, D. Angew. Chem. Int. Ed. 2005, 44, 4442-4489.

6. Parenty, A.; Moreau, X.; Niel, G.; Campagne, J.-M. Chem. Rev. 2013, 113, PR1-PR40.

7. (a) Ohtani, T.; Tsukamoto, S.; Kanda, H.; Misawa, K.; Urakawa, Y.; Fujimaki, T.; Imoto, M.; Takahashi, Y.; Takahashi, D.; Toshima, K. Org. Lett. 2010, 12, 5068-5071. (b) Dai, C.; Stephenson, C. R. J. Org. Lett. 2010, 12, 3453-3455.

8. (a) Illuminati, G.; Mandolini, L. Acc. Chem. Res. 1981, 14, 95-102. (b) Galli, C.; Mandolini, L. Eur. J. Org. Chem. 2000, 3117-3125. (c) Galli, C.; Illuminati, G.; Mandolini, L. J. Org. Chem 1980, 45, 311-315. (d) Galli, C.; Illuminati, G.; Mandolini, L.; Tamborra, P. J. Am. Chem. Soc. 1977, 99, 2591-2597. (e) Illuminati, G.; Mandolini, L.; Masci, B. J. Am. Chem. Soc. 1975, 97, 4960-4966.

9. (a) Knops, P.; Sendhoff, N.; Mekelburger, H.-B.; Vögtle, F. Top Curr. Chem. 1992, 161, 1-36. (b) Rossa, L.; Vögtle, F. Top. Curr. Chem. 1983, 113, 1-86.

10. Blankenstein, J.; Zhu, J. Eur. J. Org. Chem. 2005, 1949-1964.

11. (a) Anderson, S.; Anderson, H. L.; Sanders, J. K. M. Acc. Chem. Res. 1993, 26, 469-475. (b) Burke, S. D.; McDermott, T. S.; O'Donnell, C. J. J. Org. Chem. 1998, 63, 2715-2718. (c) Martí-Centelles, V.; Burguete, M. I.; Luis, S. V. Chem. Eur. J. 2012, 18, 2409-2422. (d) Alexander, V. Chem. Rev. 1995, 95, 273-342.

12. (a) Pérez-Vázquez, J.; Veiga, A. X.; Prado, G.; Sardina, F. J.; Paleo, M. R. Eur. J. Org. Chem. 2012, 975-987. (b) Monje, P.; Graña, P.; Paleo, M. R.; Sardina, F. J. Org. Lett. 2006, 8, 951-954.

13. (a) Donohoe, T. J.; Harji, R. R.; Cousins, R. P. C. Tetrahedron Lett. 2000, 41, 1327-1330. (b) Donohoe, T. J.; Harji, R. R.; Cousins, R. P. C. Tetrahedron Lett. 2000, 41, 1331-1334.

14. Yus, M.; Herrera, R. P.; Guijarro, A. Chem. Eur. J. 2002, 8, 2574-2584.

15. The final concentration for most experiments (performed with $0.34 \mathrm{mmol}$ of diester 1) was $0.05 \mathrm{M}$ due to experimental convenience, but when the reaction was performed at a higher scale, the final concentration was $0.1 \mathrm{M}$ and the same yield was obtained.

16. (a) The Conformational Analysis of Cyclohexenes, Cyclohexadienes and Related Hydroaromatic Compounds, Rabideau, P. W. Ed., VCH Publishers: Weinheim, 1989. (b) Rabideau, P. W. Acc. Chem. Res. 1978, 11, 141-147.

17. (a) Brinkmann, A. W.; Gordon, M.; Harvey, R. G.; Rabideau, P. W.; Stothers, J. B.; Ternay, A. L., Jr J. Am. Chem. Soc. 1970, 92, 59125916. (b) Miller, B.; Marhevka, V. C. Tetrahedron Lett. 1981, 22, 895 898. (c) Raber, D. J.; Hardee, L. E.; Rabideau, P. W.; Lipkowitz, K. B. J. Am. Chem. Soc. 1982, 104, 2843-2847.

18. ${ }^{1} \mathrm{H}$ and ${ }^{13} \mathrm{C}-\mathrm{NMR}, 2 \mathrm{D}$ HSQC and HMBC experiments on protonated $\mathbf{2 5}$ and enolate $\mathbf{2 6}$, as well as 1D NOESY on $\mathbf{2 6}$, allowed full assignment of all signals (see Supporting Information).

19. Rabideau, P. W.; Maxwell, A. J.; Sygula, A. J. Org. Chem. 1986, 51, 3181-3184.

20. (a) Kay, E. R.; Leigh, D. A.; Zerbetto, F. Angew. Chem. Int. Ed. 2007, 46, 72-191. (b) Jiang, Y.; Chen, C.-F. Eur. J. Org. Chem. 2011, 6377-6403. 